\title{
29 Transfer der Ergebnisse in ein Anwendernetzwerk
}

\section{Daniel Köhler}

Von Anfang an bestand ein zentraler Kerngedanke des optes-Projekts darin, die intern entwickelten Angebote auch der interessierten Öffentlichkeit zur Verfügung zu stellen.

Während die Dissemination der optes-Angebote innerhalb des Verbundprojekts im gegenseitigen Austausch mit den jeweils zuständigen Teilprojekten erfolgt, unterliegt die Bereitstellung dieser Angebote an die Öffentlichkeit jedoch anderen Rahmenbedingungen. Die von den einzelnen Teilprojekten entwickelten Angebote und weiteren Projektergebnisse, die teilweise für die Projektpartnerstandorte individualisiert werden, müssen in allgemeingültige, ansprechende und einfach zu handhabende Produkte für die interessierte Öffentlichkeit überführt werden. Hierunter fällt ebenfalls, das fehlende Vorwissen der interessierten Anwender*innen über Konzepte und aufeinander aufbauende Bestandteile zu berücksichtigen.

Das Teilprojekt Dissemination wurde zu diesem Zweck ins Leben gerufen, um einen gemeinsamen, qualitätsgesicherten Standard in den optes-Angeboten zu schaffen, diesen zu veröffentlichen und zu vermarkten. Die im optes-Verbundprojekt entwickelten Angebote eines jeden Teilprojektes wurden schlussendlich in der Dissemination für die Veröffentlichung gebündelt.

Als zentrale Anlaufstelle für interessierte Anwender*innen fungiert das optesAnwendernetzwerk. Über dieses auf optes.de befindliche Netzwerk kann die interessierte Anwenderschaft nach einer Registrierung über den dort befindlichen optes-Materialienpool die optes-Projektergebnisse kostenlos erwerben und andererseits über ein spezielles Anwenderforum Supportanfragen stellen sowie über Neuigkeiten rund um optes informiert werden (z. B. Versionsupdates).

\subsection{Materialienpool}

Über den Materialienpool des Anwendernetzwerkes werden die veröffentlichungsreifen Projektergebnisse vom Teilprojekt Dissemination, den auf optes.de registrierten Anwender*innen (nachfolgend auch User genannt) zur Verfügung gestellt. Um bei der Angebotsvielfalt von optes die Übersicht zu wahren und eine gute User Experience beizubehalten, werden die User im Materialienpool durch ein übersichtliches Menü mit einer stringenten Navigation geführt (siehe Abbildung 1). Nur so können die zahlreichen optes-Materialien auch von den Userin-

Die Originalversion dieses Kapitels wurde revidiert. Ein Erratum ist verfügbar unter https://doi.org/10.1007/978-3-658-31279-4_32 
Es bestehen verschiedene Zugänge zu den optes-Materialien und Angeboten auf dieser Seite - Bitte treffen Sie Ihre Auswahl:

\section{Auswahl nach dem jeweiligen optes-Angebot}

Hier können Sie alle Materialien zu den jeweiligen optes-Angeboten aufrufen.

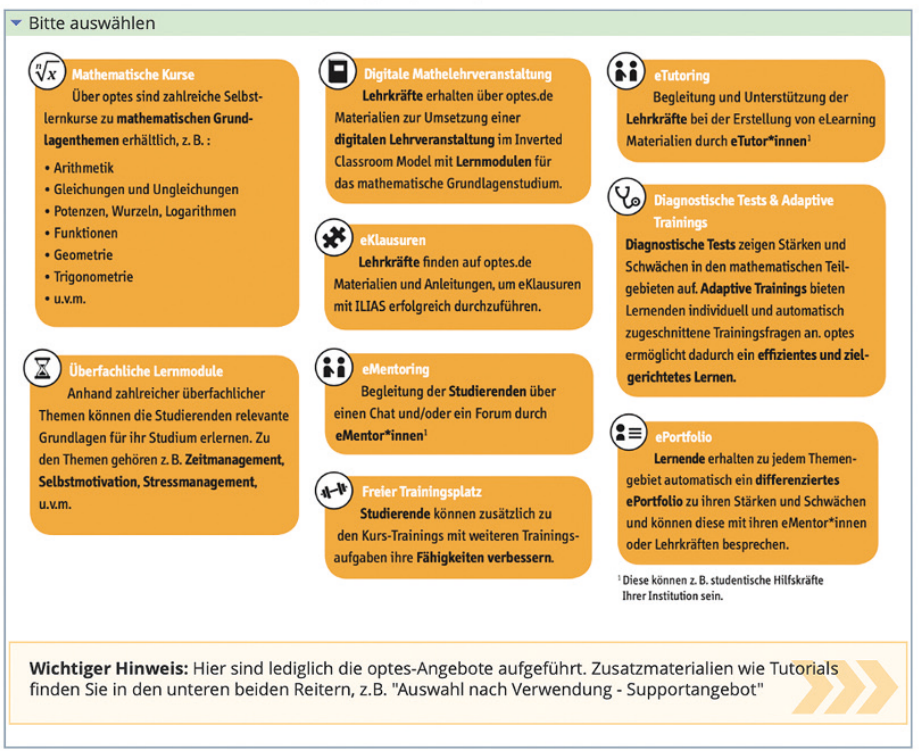

\section{Auswahl nach dem Einsatzzeitpunkt}

Beim Einsatzzeitpunkt wird angegeben, ob sich das Angebot für den Einsatz vor Studienbeginn, während der Studieneingangsphase oder im weiteren Studienverlauf eignet.

$\checkmark$ Bitte auswählen

Überfachliche Lernmodule

\section{Auswahl nach der Verwendung}

Die Sortierung nach Verwendung und Einsatzgebiet unterscheidet danach, ob Sie z.B. gezielt nach Handreichungen oder Supportangeboten suchen

$\checkmark$ Bitte auswählen

Das Projekt optes wird im Rahmen des Qualitätspakts Lehre aus Mitteln des Bundesministeriums für Bildung und Forschung unter dem

Förderkennzeichen 01PL17012 gefördert. Die Verantwortung für den Inhalt dieser Veröffentlichung liegt beim Autor.

Abbildung 1: Der optes-Materialienpool mit einer übersichtlichen und stringenten Navigation 
nen und Usern komfortabel überblickt werden, ohne durch die Angebotsfülle abgeschreckt zu werden. Darüber hinaus wurden zahlreiche optes-Angebote zu benutzerfreundlichen Paketen zusammengefasst, um den Usern das mühsame Zusammensuchen benötigter Einzelmaterialien zu ersparen.

Diese Strategie wurde vom Teilprojekt Dissemination gewählt, um insbesondere den Lehrenden von Bildungseinrichtungen, also der Zielgruppe des Teilprojekts Dissemination, gerecht zu werden. Hier gilt die Prämisse, dass Lehrende einer zeitkritischen Umgebung unterliegen und nicht die zeitlichen Ressourcen besitzen, um sich mühsam durch E-Learning-Angebote zu klicken, die noch nicht einmal Bestandteil der hiesigen Curricula bzw. Lehrpläne sind, sondern als nützliches Zusatzmaterial der eigenen Studierendenschaft zur Verfügung gestellt werden.

Mit dem Paket optes-essentials, dem Basispaket von optes, erhalten die User eine Vorauswahl an optes-Angeboten für einen schnellen und reibungslosen Einstieg. Mit optes-essentials + werden eine größere Auswahl an optes-Angeboten und weitere Zusatzmaterialien bereitgestellt. Jedes Paket enthält außerdem eine Installationsanleitung und demnächst auch noch ein Benutzerhandbuch, dass wie eine Art Bedienungsanleitung fungieren soll. Diese Strategie, den Usern eine Vorauswahl an optes-Angeboten in Form von Paketen anzubieten, erhöhte nach Rücksprache mit einigen Anwender*innen die Annahme der optes-Angebote beträchtlich. Gleichzeitig muss an dieser Stelle ebenfalls betont werden, dass mit den optes-Paketen erstmalig in sich geschlossene Selbstlernkurse, bestehend aus weiteren Teilmaterialien, angeboten wurden. Zuvor existierten lediglich die jeweiligen Teilmaterialien, die von den Usern erst noch in ihrer ILIAS-Installation zu eigenen Selbstlernkursen zusammengefügt werden mussten. Die nun von optes bereitgestellten Selbstlernkurse erhöhen den Komfort für die Anwenderschaft enorm.

Neben der Überführung der optes-Angebote in veröffentlichungsreife Produkte wurde vom Teilprojekt Dissemination sowie einzelnen optes-Teilprojekten für die Anwendenden ebenfalls eine Reihe an Zusatzmaterialien erstellt, um die den optesAngeboten zugrundeliegenden Konzepte und didaktischen Überlegungen zu erläutern. Konkret waren dies animierte Erklärvideos, Screencasts, Anleitungen sowie eigene Lernmodule, um die Welt von optes bzw. erste Schritte in ILIAS kennenzulernen und zu überblicken.

\subsection{Anwenderforum}

Über das Anwenderforum konnten die registrierten User Fragen an optes stellen und sich über Neuigkeiten rund um optes informieren. Das Anwenderforum ist das zentrale Kommunikationsinstrument zwischen optes und der Anwenderschaft. Ferner ist das Anwenderforum dazu gedacht, dass sich die User untereinander über das Forum zu einer Community vernetzen und sich gegenseitig austauschen bzw. unterstützen. Diesem Vernetzen der Anwendenden zu einer Community liegt die 
Idee zugrunde, dass optes von dieser Community über das Projektende hinaus getragen und mit Neuerungen weiterentwickelt wird.

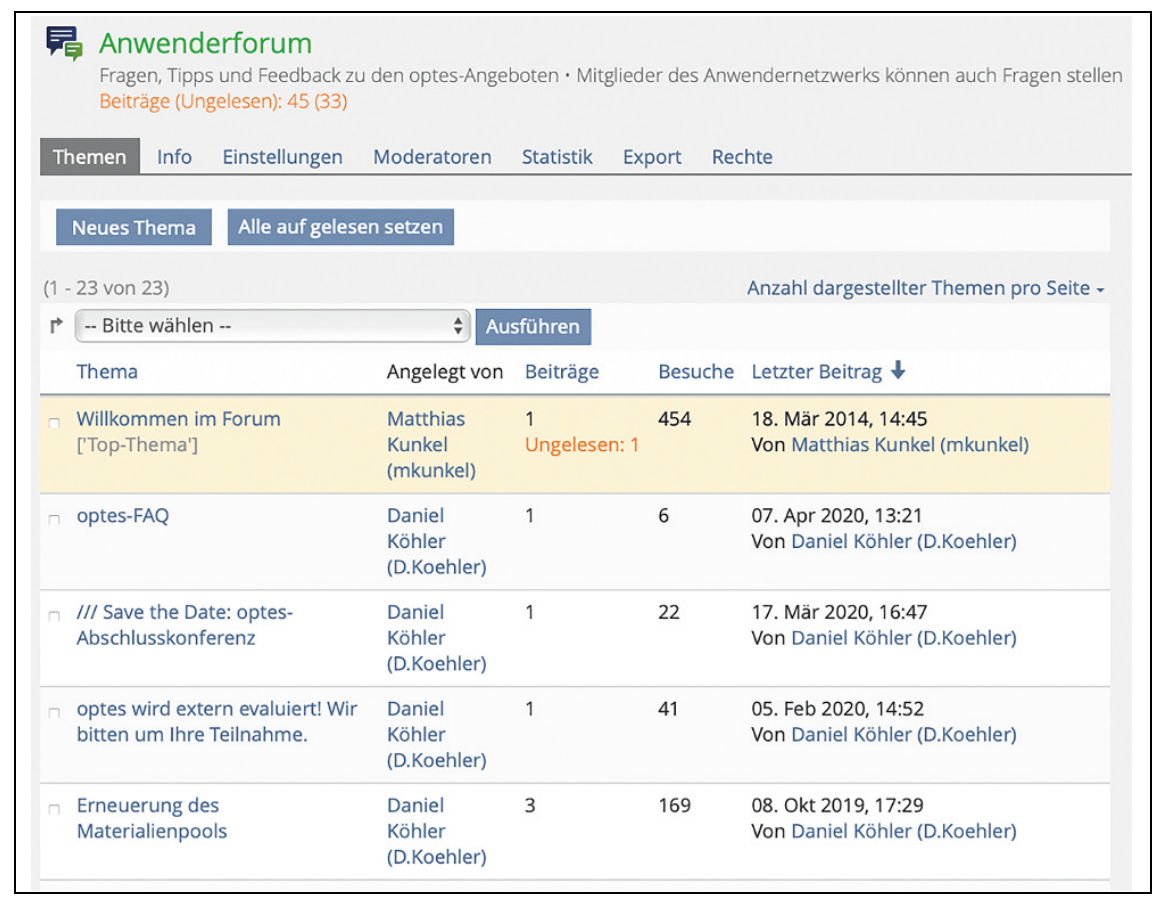

Abbildung 2: Das optes-Anwenderforum

Von der Anwendercommunity können die optes-Angebote beispielsweise kontextualisiert, abgeändert oder ergänzt und anderen Usern zur Verfügung gestellt werden. Ein Beispiel hierfür sind Schulen: Bedingt durch einen großen Zulauf an Schulen, die sich stark für die auf die Hochschulen ausgerichteten optes-Angebote interessieren, aber nicht die eigenen Ressourcen besitzen, diese Inhalte auf den Schulkontext anzupassen, besteht die Möglichkeit, sich über das Anwenderforum eigenständig mit anderen Anwender*innen zu organisieren und darüber die Ressourcen untereinander aufzuteilen. Jedoch wurde das Anwenderforum für diesen Zweck bislang weder von den Schulen, noch von anderen Anwender*innen genutzt. Für das rege Funktionieren einer selbsttragenden Community ist aber sicherlich entscheidend, dass sich die Anwenderschaft zuvor entsprechend intensiv mit den optes-Angeboten auseinandergesetzt, bzw. diese erst eine gewisse Zeit im Einsatz hatte und darüber hinaus auch die hierfür erforderlichen zeitlichen Ressourcen besitzt. 
Für eine erfolgreiche Dissemination der optes-Angebote an externe Bildungseinrichtungen wurden folgende Rahmenbedingen definiert, die den Erfolg der Dissemination gewährleisten sollen:

(1) Nachhaltigkeit und Dezentralisierung der Angebote (essenzieller Schlüsselfaktor)

Die Gewährleistung eines nachhaltigen Einsatzes der optes-Angebote ist ein entscheidender Garant für eine erfolgsversprechende und langfristig wirkungsvolle Dissemination. Daher hatte man sich im optes-Projekt für zwei Wege der Nachhaltigkeit entschieden:

- Die optes-Angebote sollen lokal auf den jeweiligen Hochschulservern der teilnehmenden Hochschulen unabhängig von optes.de funktionieren. So wird sichergestellt, dass bei einem etwaigen Projektende die optes-Angebote weiterhin von den Hochschulen genutzt werden können. Dadurch entschied man sich bei optes für das Lernmanagementsystem ILIAS, da dieses hierfür nicht nur die geeignete technische Infrastruktur bietet - auch über das Projektende hinaus , sondern ebenfalls

- vielschichtige Individualisierungs- und Anpassungsmöglichkeiten erlaubt. Somit können die optes-Angebote systemseitig durch ILIAS individualisiert und angepasst werden. Damit sind nicht nur weitreichende gestalterische Anpassungen der Kurse oder des Designs, sondern ebenfalls inhaltliche Aktualisierungen (z. B. von Fragenpools) möglich. Diese Möglichkeit der Aktualisierung und Anpassung gewährleistet die zweite Dimension der Nachhaltigkeitsstrategie von optes.

Diese beiden Dimensionen werden vom Teilprojekt Dissemination nach wie vor als absolut essenziell für eine erfolgreiche und nachhaltige Dissemination der optes-Angebote eingestuft.

\section{(2) Solider rechtlicher Rahmen (essenzieller Schlüsselfaktor)}

- Angesichts der strengen Datenschutzgrundverordnung (DSGVO) bestehen verständlicherweise oftmals Unsicherheiten über den Einsatz von Dienstleistungen aller Art. Damit projektexterne Bildungseinrichtungen in keinerlei rechtliche Problemstellungen oder Unsicherheiten geraten und sich infolgedessen gegen optes entscheiden, wird den Bildungseinrichtungen von optes ein attraktives Lizenzmodell zur Verfügung gestellt. Alle veröffentlichten optesProjektergebnisse, also auch die optes-Angebote, unterliegen fest definierten und für beide Seiten praktikablen Creative-Commons-Lizenzen. Für die meisten optes-Projektergebnisse wurde die Lizenz CC BY-SA gewählt, die einerseits die Autorenschaft schützt und gleichzeitig eine Vervielfältigung, Abänderung, sowie kommerzielle Nutzung (z. B. Nutzungsentgelte, Einsatz an 
privaten Hochschulen) gestattet. Die CC-Lizenzen sind klar definiert und unter den Open Education Resources stark verbreitet. Diese klare rechtliche Regelung wird vom Teilprojekt Dissemination ebenfalls als essenziell für eine erfolgreiche und nachhaltige Verbreitung der optes-Angebote betrachtet.

\section{(3) Öffentlichkeitsarbeit (essenzieller Schlüsselfaktor)}

- Damit die optes-Angebote auch der interessierten Öffentlichkeit zur Verfügung gestellt werden konnten, bestand eine der Hauptaufgaben des Teilprojekts Dissemination darin, das Anwendernetzwerk aufzubauen, um dann schlussendlich über das Netzwerk die optes-Angebote zu veröffentlichen.

- Über das Teilprojekt Dissemination wurde die Strategie verfolgt, in einem ersten Schritt auf optes aufmerksam zu machen, um dann in einem zweiten Schritt Informationsveranstaltungen bei den interessierten Hochschulen vor Ort anzubahnen. Durch diese Veranstaltungen sollten die optes-Angebote schlussendlich bei den interessierten Hochschulen zum Einsatz gebracht werden. Interessierte Hochschulen konnten die optes-Angebote jedoch auch ohne Informationsveranstaltungen jederzeit über den Materialienpool beziehen. Dies ist bei dem mittlerweile über 200 Anwender*innen umfassenden Anwendernetzwerk vermutlich häufig geschehen.

- Für den ersten Schritt, die Publikmachung, wurden verschiedene vertriebsund marketingstrategische Maßnahmen ergriffen. Diese bestanden etwa im Versand von professionellen Werbematerialien in digitaler und Printform, Telefonakquisen, Präsentationen auf ILIAS-Treffen sowie Auftritten auf einer E-Learning-Messe. Aber auch die zahlreichen Präsentationen auf Qualitätspakt-Lehre-Veranstaltungen oder Symposien durch Mitarbeitende des optesProjekts führten zur Publikmachung von optes.

- Am erfolgreichsten von diesen Maßnahmen erscheinen jedoch die direkten Telefonakquisen, über die Lehrbeauftragte direkt angesprochen wurden, sowie Präsentationen auf ILIAS-Veranstaltungen. Letzteres führte vermutlich deshalb zum Erfolg, da hier naturgemäß zahlreiche Hochschulen vertreten sind, die ILIAS bereits im Einsatz haben und dadurch offenbar eine entsprechende Affinität für die Annahme von ILIAS-basierenden E-Learning-Angeboten aufweisen. Aber auch die Präsentation der optes-Angebote auf einschlägigen Veranstaltungen durch optes-Projektmitarbeitende stellte sich als erfolgreich heraus.

- Da von optes nicht erfasst wird, welche Angebote von welchen Nutzern bezogen werden, ist es letztendlich schwierig zu bewerten, welche Maßnahmen schlussendlich entscheidend bzw. weniger entscheidend für die Dissemination der optes-Angebote waren. Zwar versuchte das Teilprojekt Dissemination über verschiedene Wege, Anwenderfeedbacks einzuholen, doch war grundsätzlich die Bereitschaft für Feedbacks innerhalb der Anwenderschaft äußerst gering. 
Die Herangehensweise, Informationsveranstaltungen bei interessierten Hochschulen durchzuführen, bewährte sich ebenfalls, da durch diese Maßnahme auf individuelle Fragestellungen eingegangen werden konnte und sich alle beteiligten Akteure intensiv mit der Thematik beschäftigten. Vermutlich eröffneten die Informationsveranstaltungen den besten Zugang zur Zielgruppe des Teilprojekts Dissemination, den Hochschuldozent*innen, da sich diese nicht erst in die Thematik einlesen mussten. Außerdem wurden über die Veranstaltungen auf einen Schlag gleich mehrere Dozent*innen informiert und überzeugt, da auf den Veranstaltungen meist das gesamte Kollegium vertreten war - auch die Lehrenden, die dem ELearning im Allgemeinen eher skeptisch gegenüber stehen. Über Live-Demos konnte durch das Aufzeigen von interaktiven Animationen schnell der Mehrwert von E-Learning-Angeboten gerade im Bereich der Mathematik aufgezeigt werden, sodass die Skeptiker*innen den optes-Angeboten schlussendlich enthusiastisch gegenüberstanden. Teilweise kam dann sogar die Frage auf, weshalb solche Angebote bislang noch nicht eingesetzt wurden. Insgesamt waren Informationsveranstaltungen also eine sehr effektive und zielgerichtete vertriebs- und marketingstrategische Maßnahme.

Die Dauer bis zum Einsatz der optes-Angebote durch die Hochschulen nach diesen Informationsveranstaltungen betrug wenige Tage bis hin zu über sechs Monaten.

- Die Öffentlichkeitsarbeit/Marketingmaßnahmen als solche werden vom Teilprojekt Dissemination ebenfalls als essenziell für eine erfolgreiche und nachhaltige Verbreitung der optes-Angebote eingestuft. Es reicht heutzutage durch die Dichte an Informationen und Angeboten - auch bei Projekten aus dem Qualitätspakt Lehre - längst nicht mehr aus, Material einfach nur einer breiten Masse zur Verfügung zu stellen. Multiplikator*innen müssen identifiziert bzw. geschaffen werden, um die Zielgruppen zuverlässig mit den Angeboten erreichen zu können.

\section{$29.3 \quad$ Resümee}

Damit auch externe Bildungseinrichtungen einen Nutzen von den erstellten optesAngeboten haben, wurden diese vom Teilprojekt Dissemination in Abstimmung mit den anderen optes-Teilprojekten auf einen Qualitätsstandard gebracht und über den Materialienpool des Anwendernetzwerks veröffentlicht.

Über das Anwenderforum, die zweite Achse des Anwendernetzwerks, können sich die registrierten Anwender*innen zu einer Community zusammenschließen, untereinander austauschen, sowie Anfragen an optes stellen und von optes ebenso über Neuigkeiten informiert werden. Das Anwenderforum fungiert als die zentrale Kommunikationsplattform zwischen optes und der externen Anwenderschaft.

Das Teilprojekt Dissemination kümmerte sich ebenfalls um den Aufbau des Anwendernetzwerkes. Über verschiedene vertriebs- und marketingstrategische 
Maßnahmen wurde dieses Netzwerk aufgebaut. Im ersten Schritt wurde über den Versand von professionellem Informationsmaterial per Post oder E-Mail, Telefonakquisen, Messeauftritten, sowie Präsentationen auf ILIAS-Treffen auf optes aufmerksam gemacht. Aber auch auf Treffen des Qualitätspaktes Lehre oder speziellen Symposien berichteten optes-Mitarbeitende anderer Teilprojekte von optes. Unter den Bildungseinrichtungen, die optes-Angebote nutzen und der optes-Dissemination bekannt sind, waren die Telefonakquise und optes-Präsentationen auf ILIAS-Treffen besonders erfolgreich. Aber auch das Präsentieren auf einschlägigen Veranstaltungen, wie den QPL-Treffen oder Symposien, führte zu guten Resonanzen. Im zweiten Schritt sollten die Hochschulen über Informationsveranstaltungen vor Ort schlussendlich von optes überzeugt werden.

Diese Herangehensweise bewährte sich, da gerade auf den Informationsveranstaltungen vor Ort besonders skeptische Lehrende anhand von Live-Demos schnell überzeugt werden konnten. Gerade das Vorführen von interaktiven Animationen zeigte klar den Mehrwert von E-Learning-Angeboten auf. Bis zum Einsatz der optes-Angebote an den Hochschulen vergingen in der Regel zahlreiche Monate. Die Vertriebs- und Marketingmaßnahmen bzw. Öffentlichkeitsarbeit waren für eine erfolgreiche Verbreitung der optes-Angebote unerlässlich.

Open Access Dieses Kapitel wird unter der Creative Commons Namensnennung 4.0 International Lizenz (http://creativecommons.org/licenses/by/4.0/deed. de) veröffentlicht, welche die Nutzung, Vervielfältigung, Bearbeitung, Verbreitung und Wiedergabe in jeglichem Medium und Format erlaubt, sofern Sie den/ die ursprünglichen Autor(en) und die Quelle ordnungsgemäß nennen, einen Link zur Creative Commons Lizenz beifügen und angeben, ob Änderungen vorgenommen wurden.

Die in diesem Kapitel enthaltenen Bilder und sonstiges Drittmaterial unterliegen ebenfalls der genannten Creative Commons Lizenz, sofern sich aus der Abbildungslegende nichts anderes ergibt. Sofern das betreffende Material nicht unter der genannten Creative Commons Lizenz steht und die betreffende Handlung nicht nach gesetzlichen Vorschriften erlaubt ist, ist für die oben aufgeführten Weiterverwendungen des Materials die Einwilligung des jeweiligen Rechteinhabers einzuholen.

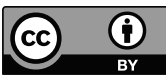

